\title{
UAV Tomographic Synthetic Aperture Radar for Landmine Detection
}

\author{
Muhannad Almutiry \\ Electrical Engineering Department \\ Northern Border University \\ Arar, Saudi Arabia \\ muhannad.almutiry@nbu.edu.sa
}

\begin{abstract}
The development of the Unmanned Aerial Vehicle (UAV) and communication systems contributed to the availability of more applications using UAVs in military and civilians purposes. Anti-personnel landmines deployed by militia groups in conflict zon es are a life threat for civilians and need cautious handling while removing. The UAV Tomographic Synthetic Aperture Radar (TSAR) can reconstruct three-dimension images of the investigation domain to prescreen nonmetallic landmines. A nonmetallic landmine cannot be detected using conventional ground penetrating radars when the scattering field is undetected due to the dielectric permittivity. In this paper, imaging the underground for detecting landmine using TSAR is proposed. The TSAR has the capability of prosing the data in discrete mode regardless of the altitude of UAV's radar. A landmine is always buried less than a feet depth. L-band frequency is used to provide high resolution and to penetrate deep in dry soil. More than one UAVs are used to multistatic scan the investigation space. The geometric diversity of multistatic distribution of the sensors will provide more information about the buried nonmetallic landmines, certain features, and their location. The data collected from the sensors will align with the geolocation data obtained from the UAV's system for processing. Dynamic flying can be used to predict the electromagnetic response of the scattering field to create a dynamic matching filter using the Green's function under first-order Born approximation. The occurring air-soil interference has been removed as an unwanted reflection from the ground while keeping the signal coming from underground. Using the Born approximation assumption created an ill-posed linear system solved by the Conjugate Gradient algorithm. Simulation results are presented to validate the method.
\end{abstract} radar

Keywords-radar; RF tomography; $U A V$; synthetic aperture

\section{INTRODUCTION}

There were an estimated one hundred million landmines positioned in many countries by 2009 , while their number is increasing by 2 million landmines annually $[1,2]$. These hidden weapons pose a serious threat to the civilians living in these regions. While efforts are been conducted to remove these mines, at the ongoing rate of clearance it will take an estimated 1100 years to eradicate them and perform ordinances of unexploded mines through the use of conventional methods of detection such as Ground-Penetrating Radar (GPR) [3].
TSAR exhibits three primary attributes that set it apart from alternative sensors: a wide accumulated angle, low-frequency band, and small bandwidth. These attributes facilitate a more effective penetration of the soil, the development of Synthetic Aperture Radar (SAR) images that are of a higher resolution, and enable the radar to cover more extensive areas. As such, TSAR represents a promising technology for the ongoing efforts to find land mines from a safe distance. Moreover, tomography has been effectively employed in many medical and scientific fields. For example, Computed Tomography (CT) is frequently used to assist diagnosis. The tomographic set of scanning is exploiting the spatial diversity as an X-ray beam is concentrated and scanning in multiple angles around the object is conducted in order to provide the 3-D features [4]. The attenuation that occurs as the object is passed is calculated by the detectors (X-ray receivers) that are facing the X-ray tube. At each point at the circular geometry, the radiation dose that is received relates to an estimation of the attenuation of the object in the direction of the transmission. According to the projection-slice theorem [5] for a given angle position the Fourier transform of the signal that is subsequently received relates to a slice of the two-dimensional (2-D) Fourier transform. During the process of the CT, a one-dimensional (1D) FFT is first performed. This is then processed into a 2-D image that displays the distribution of the attenuation in a polar arrangement. After that, the polar format is transformed into a Cartesian format via an interpolation step. The final image is developed via a 2-D inverse that results in the presentation of the 2-D object attenuation map at every position of interest.

Several studies [6-8] have demonstrated that it is possible to formulate spotlight SAR spectral analysis based on azimuth in a comparable technique similar to the tomographic set of medical X-ray scanning $[9,10]$. The radar tomographic set is based on linking the radars in one processing network, where it can be expanded to further application $[11,12]$. Furthermore, the stripmap mode can be performed using a similar process to tomographic processing based on the scanning trajectory [13]. As a result of the small variations in the angles that are of interest during SAR processing, more algorithms have been developed for effective image reconstruction [14]. At a high level, the development of the synthetic aperture that is employed in SAR represents a tomographic technique. When $\mathrm{X}$-ray tomography is performed, it is not possible to achieve 3- 
D imaging. Instead, 2-D processing is performed in iterations spanning multiple positions on the object by performing minor step parallel readings of the receiver and transmitter set. This form of CT can be applied to detect mines due to the mutlistatic tomographic radar that can process signals at any evaluation beam level, where a discrimination process can be applied to classify a landmine from a variety of objects at a selected area that is potentially prescreening. Having the ability to detect and image objects that are buried in the ground is essential in a range of commercial, military, and civilian settings, i.e. for the exploration of natural resources and detection of tunnels [15].

Below-ground imaging techniques that are currently in use have the ability to identify both metallic and nonmetallic artifacts that exhibit higher conductivity than soil $[16,17]$. The use of electromagnetic waves to image objects that are located below the ground represents a non-damaging approach to the detection, surveillance, and imaging of below-ground features and irregularities. The performance of below-ground imaging is performed using a process of radio wave transmission and scattering by which radio waves are passed through the ground by the transmitter system, which subsequently measures the waves that are reflected from the targets and determines the effects that the various materials have on the transmitted radio waves. The data that are reflected from the area of interest are primarily received by passing a surface antenna around a circular framework. Pulses are transmitted from the airborne UAV's radar downward-looking to the ground with a data link connection for effective and accurate processing. The main difficulty in this scenario is the capturing of the signal caused by reflections of the surface. When the targets are deeply buried, it is easy to separate the surface clutter signal from the target signal. In such cases, it is easy to separate signals using range gating techniques. If the target is very close to the surface, range gating is ineffective because the clutter signal from the surface and the target will be received almost at the same time. Thus, the problem is to promote the separation of target signals and ground clutter. TSAR can operate at UltraNarrow Bandwidth (UNB) or even at a single frequency in pulse form to reduce the attenuation when these pulses penetrate the ground and come into contact with an underground landmine. The signal attenuation is associated with the bandwidth. The radar system employs a supercomputer to undertake real-time digital processing of the scattering data field collected. When covering large environments, the data rate is substantial, and so a single receiver system using a single frequency is required for realtime processing employing existing down-link hardware.

The surface antenna assembles the scattering field data that are reflected and subsequently undergo processing for detection and imaging. Dynamic Green's function is used to eliminate the strong surface clutter. In Dynamic Green's function technique, the processing of the landmine imaging is done by using just a single frequency. The technique explores an image of a below-ground item that is reconstructed through the application of TSAR and 3-D SAR using matched filter calculations based on aviation data that permit the collection of circular data collection.

\section{LAND MINE DETECTION USING ELECTROMAGNETICS}

Land mines are currently detected through the use of metal detectors that evaluate the disruption of a radiated electromagnetic field from the presence of underground metallic objects. One of the primary drawbacks of this approach is that any scrap metal activates the alarm. As such, it represents an inefficient approach to the detection of land mines due to the high rate of false alarms [18]. The GPR is used to transmit electromagnetic waves that penetrate the ground to sense the underground from the reflections that occur at the discontinuities of the dielectric constant. Due to its ability to penetrate the ground, Ultra-Wide Band Synthetic Aperture Radar (UWB SAR) has emerged as a promising technology that can identify landmines in a large area of land without putting human lives at risk [19-20]. However, like metal detectors, GPR can trigger false alarms due to the presence of irregularities in the soil, for instance, rocks and roots, when hidden Markov models were used to classify the background from plastic-cased or completely nonmetal landmines [21-23]. A further issue with GPR is that it is not as effective in terms of detection of smaller mines that are in shallow locations because the soil-surface reflection disguises their response $[1,24]$. The $\mathrm{X}$-ray backscattering approach is based on the notion that soil and mines have different attenuation, which leads to detecting landmines [25] although the x-ray generators that are employed in this approach are huge and heavy and require high amounts of power to achieve sufficient penetration. As such, this does not represent a portable method [26]. Furthermore, as radiation is involved, its acceptance is limited. The millimeter-wave radar (MMWR) approach is dependent on the concept that the soil has low reflectivity and high emissivity at certain frequencies, while metals have the opposite characteristics [27, 28]. Active MMWR employs a source of excitation, but MMWR relies purely on the temperature of the environment. As such, while MMWR represents a promising approach for the detection of metallic objects, it is not effective in the detection of plastic artifacts.

\section{RELATED WORK}

A number of methods of signal processing have been put forward for improving landmine GPR system performance, including forward scattering radar classification [29], background subtraction, CLEAN algorithm [30], Kalman filters [31], likelihood ratio test [32], wavelet packet decomposition [33], and additional two-dimensional filtering [34]. The majority of these methods depends on estimating the background signal through Green's function or calculating a mean value for the unprocessed data collected by GPR and then subtracting the estimated background signal from the received signal. Such methods have been widely used for GPR applications but are a compromise at best.

An alternative approach that does not need background data has been suggested in [35]: rather than being dependent on background scene data, we can gate out ground reflections, estimate the corresponding parameters, and use this for modeling and subtracting wall contributions from the received data. GPR designed for the detection of buried landmines through multiple probes of the ground across the target area has a similar problem caused by clutter reflection, i.e. reflection 
from the ground's surface. This approach involves the application of matched filters for eliminating ground clutter, which can be estimated at the phase center of the scattering field. While GPR requires special filtering, these two challenges have two substantial differences. Firstly, for GPR, clutter signals arise from echoes caused by the air/soil medium differences. This means the strong ground clutter will mask the landmines which are very close to the surface. Those buried deep down will not be shielded by clutter. Secondly, as the clutter/landmine scatting signal is significantly overlapped within frequency domains, filtering will also attenuate the landmine reflection. For numerous GPR applications, the inverse problem of imaging a whole medium is not practical. The inverse problem will generally be nonlinear even when the forward problem is linear. Additionally, the inverse problem is generally ill-conditioned, and we have to apply an inversion technique to regularize the solution. In this case, we employ techniques centered in matched filter methodology based on dynamic Green's function for the detection of significant changes in patterns within the cluttered environment, e.g. the appearance/disappearance of targets/target motion, with no knowledge of the background medium.

\section{Methodology}

The proposed dynamic Green's function is based on RF tomography imaging introduced at $[15,36]$. The TSAR model incorporates an electromagnetics source that is mounted into a UAV located at an instant time at $r_{m}^{t}$. The instant time can be determined by combining the information from the Global Positioning System (GPS) and the radar signal to determine the exact distance form the ground. There are many methods to support accurate fly level information, such as Lidar. The electromagnetic field radiates toward the ground which is known as the $E^{i n}$ incident field in terms of the target. The area of interest that needs to be constructed for the image is discretized where more than one UAV can scan. The target is positioned at $r_{p}$ about $0.1 \mathrm{~m}$ under the strong ground clutter, and the scattering field $E^{S C}$ is comparable to the way of radiating from the airborne UAV transmitter. This scattered field, which is known as $E^{S C}$, is recorded by a receiver at $r_{m}^{r}$. The $E^{i n}$ and $E^{S C}$ will have the same polarization unless the target has no properties of changing the reflection polarization. Additionally, each respective object is assumed to be isotropic due to the ground environment where the $E^{i n}$ and $E^{S C}$ together form the total electric field $E^{\text {tot }}$ as:

$$
E^{t o t}=E^{S C}+E^{\text {in }}
$$

The incident field can be formed as Green's function with knowing the aviation and sensor parameters to given $r_{n}^{t}, r_{m}^{r}$, and $r_{p}$ as follows:

$$
E^{i n}=j \omega \mu G\left(r_{n}^{t}, r_{p}\right)
$$

The Green's function $G\left(r_{n}^{t}, r_{p}\right)$ is giving the calculated electromagnetic response at any point in the area of interest with respect to $r_{p}$, and $k$ is the wavelength number:

$$
G\left(r_{n}^{t}, r_{p}\right)=\left(I+\frac{\nabla \nabla}{k^{2}}\right) \frac{e^{j k\left\|r_{n}^{t}-r_{p}\right\|}}{4 \pi\left\|r_{n}^{t}-r_{p}\right\|}
$$

In order to obtain the dynamic Green's function to remove strong air-soil clutter, the propagation number needs to be updated due to the soil dielectric properties. Now, we calculate the ground clutter respace above the area of interest $r_{p c}$ as:

$$
r_{p c}=\frac{c}{2 P F R}
$$

where $P R F$ is Pulse Repetition Frequency and $c$ is the speed of light.

The Green's function to calculate the clutter $G^{c}\left(r_{n}^{t}, r_{p}\right)$ is giving the calculated electromagnetic response at any point in the area of interest with respect to $r_{p c}$ :

$$
G^{c}\left(r_{p c}, r_{m}^{r}\right)=\left(I+\frac{\nabla \nabla}{k^{2}}\right) \frac{e^{j k\left\|r_{p c}-r_{m}^{r}\right\|}}{4 \pi\left\|r_{p c}-r_{m}^{r}\right\|}
$$

The scattered field can be obtained in the integral form of respect to the object function $v\left(r_{p}\right)$ and the total electric field $E^{\text {tot }}$ as follows:

$$
E^{s c}\left(\omega, r_{n}^{t}, r_{m}^{r}\right)=\iiint G\left(r_{p}, r_{m}^{r}\right) v\left(r_{p}\right) \mathrm{E}^{t o t}\left(\omega, r_{p}\right) d r_{p}
$$

It is not possible to solve the scattered field, $E^{S C}$, from (5) because it is incorporated in $E^{\text {tot }}$ and becomes a nonlinear integral equation, as outlined in (1). The incident field can be calculated using (4) and by applying the first-order Born approximation to linearize. In addition, $v\left(r_{p}\right)$ is an unknown element within the imaging problem. It is multiplied by the other unknown, $E^{S C}$ that is incorporated in the $E^{\text {tot }}$. It is possible to apply the Born approximation to the total electric field $E^{\text {tot }}$ to substitute the incidence field $E^{i n}$ to linearize the integral equation as:

$$
E^{s c}\left(\omega, r_{n}^{t}, r_{m}^{r}\right)=\iiint G\left(r_{p}, r_{m}^{r}\right) v\left(r_{p}\right) \mathrm{E}^{i n}\left(\omega, r_{p}\right) d r_{p}
$$

The incident field can be substituted with (2) to produce:

$$
E^{s c}\left(\omega, r_{n}^{t}, r_{m}^{r}\right)=\iiint G\left(r_{n}^{t}, r_{p}\right) G\left(r_{p}, r_{m}^{r}\right) v\left(r_{p}\right) d r_{p}
$$

We need now to update the Green's function in the integral by the clutter Green's function:

$$
E^{s c}\left(\omega, r_{n}^{t}, r_{m}^{r}\right)=\iiint G^{c}\left(r_{p c}, r_{m}^{r}\right) G\left(r_{n}^{t}, r_{p}\right) G\left(r_{p}, r_{m}^{r}\right) v\left(r_{p}\right) d r_{p}
$$

Discretizing (8) to have more flexibility to be adopted into airborne processing while calculating the Green's function as we collect the scattering field in different aviation level and trajectory by dividing the area of interest into $p$ pixels as follows:

$$
\begin{gathered}
E^{s c}\left(\omega, r_{n}^{t}, r_{m}^{r}\right) \cong L\left[v\left(r_{p}\right)\right] \cong \\
\sum_{p=1}^{1}\left\{G^{c}\left(r_{p c}, r_{m}^{r}\right) G\left(r_{n}^{t}, r_{p}\right) G\left(r_{p}, r_{m}^{r}\right) v\left(r_{p}\right)\right.
\end{gathered}
$$

Equation (9) can be mathematically considered in a matrix form to provide an effective and rapid process in digital signal processing in form:

$$
E_{n m}=L_{n m p} \cdot v
$$

To obtain the object function $v$, we will solve (10) as an inverse problem that is an ill-posed and ill-conditioned linear system representing the forward scattering model. However, (9) can only be used to determine single measurements as it relates to a particular orientation and transmitter location, 
receiver orientation location, orientation, and frequency. In the event any of these parameters are reformed, there is a requirement to obtain a new measurement. As such, there is a need to modify (9) in response to any variations by compiling a set of $(q=1, Q)$ airborne UAV's measurements for each divided pixel $p$ as $[8,9]$ :

$$
\begin{aligned}
e_{q p}= & l_{q p}^{x x} v_{q p}^{x x}+l_{q p}^{x y} v_{q p}^{x y}+l_{q p}^{X z} v_{q p}^{x z}+\cdots \\
+l_{q p}^{y x} v_{q p}^{y x}+ & +l_{q p}^{y y} v_{q p}^{y y}+l_{q p}^{y z} v_{q p}^{y z}+\cdots \\
& +l_{q p}^{z x} v_{q p}^{z x}+l_{q p}^{z y} v_{q p}^{z y}+l_{q p}^{z z} v_{q p}^{z z}
\end{aligned}
$$

Each $l$ value in (11) can be calculated by appropriately reorganizing and modifying (10). Expanding the equation outlined above to all pixels in the area of interest and all potential measurement patterns, $Q$, we have:

$$
\begin{aligned}
& \begin{array}{cc}
\begin{array}{c}
\text { Measurement } \\
\text { Vector } \\
(\text { Scattered Field })
\end{array} \quad \text { Dynamic Green's Function (Matching Filter) } & \begin{array}{c}
\text { Contrast } \\
\text { Vector } \\
\text { (Image) }
\end{array}
\end{array}
\end{aligned}
$$

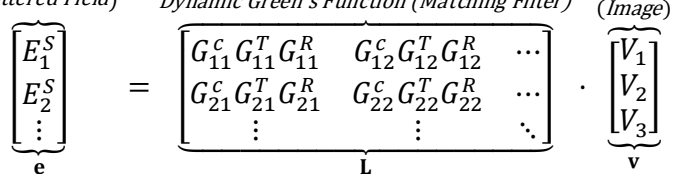

where $\mathbf{e}$ is the measured scattering field data collated at various UAV's aviation level, the matched filter $\mathbf{L}$ is the calculated Green's function response for the transmitter and the receiver at each $p$ pixel in the area of interest grid, and $\mathbf{v}$ is the unknown object function. The inversion technique to recover the object function is given by:

$$
\mathbf{v}=\mathbf{L}^{-1} \mathbf{e}
$$

The inversion of the ill-posed matrix at (12) can be obtained using the Conjugate Gradient (CG) algorithm, which is much faster compared to other inversion algorithms such as Algebraic Reconstruction Techniques (ART). The inversion algorithm can be done after the prescanning of the area of interest to do offline processing. Real-time processing is doable due to independent measurement in discrete form analysis, where the pixels of the area of interest are updated at each scan.

\section{Simulation RESUlts}

The simulation of the landmine detection using UAV TSAR has been done using FEKO, which is a computational EM simulation software tool. In this simulation, we first need to check the accuracy of the calculated dynamic Green's function, whether it really can estimate the ground clutter or not in order to update the matched filter. In Figure 1, we calculated the scattering field at using the same parameters for Green's function and FEKO simulation, which appear matching at the phase and amplitude of the reflected signal. The matching between the calculated and simulated scattering field indicates an exact update for the dynamic Green's function in the system to predict the effect of the clutter in the received signal. The accuracy of the calculated Green's function will eliminate the clutter by updating the matched filter. Furthermore, obtaining the range distance between the UAV and the ground improves the accuracy of the calculated clutter response at the matching filter. We are using the simulated radar scattering single received at L-band $500 \mathrm{MHz}$ bandwidth to obtain the range distance. The exact range distance of the radar and the measured range distance are shown in Figure 2, which can provide the accurate calculation of the clutter response using the Green's function. We placed the radar first at $2.4 \mathrm{~m}$ then we measured the range distance by the received scattering field. Then, we increased the aviation level from the ground by $0.4 \mathrm{~m}$ to find if the measured range is matching the flying level. As shown in Figure 2, the flying level was increased by $0.4 \mathrm{~m}$ for each position and the measured radar range matched the exact range. For landmine imaging, we show two scenarios with the same number of sensors at two different flying patterns. The operation frequency of both scenarios was at $2 \mathrm{GHz}$.

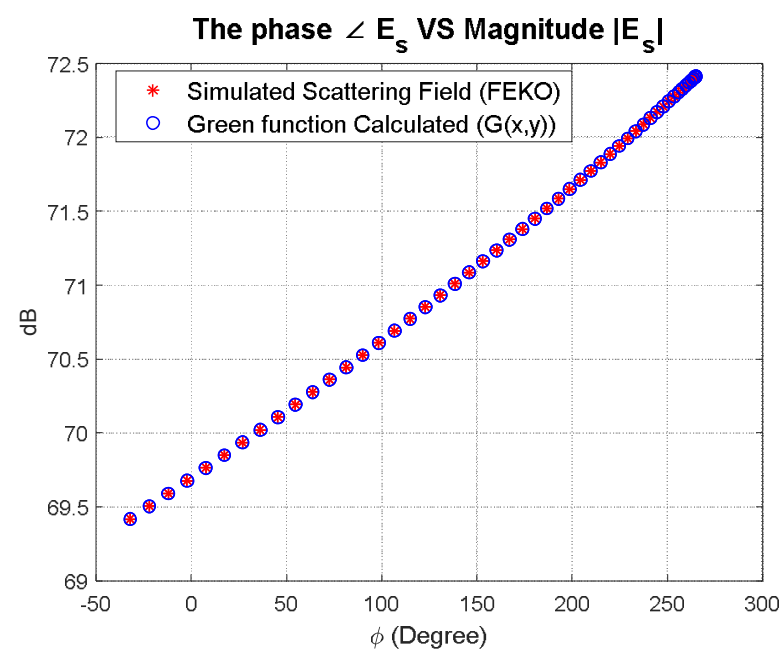

Fig. 1. Comparing the accuracy of the calculated scattering field.

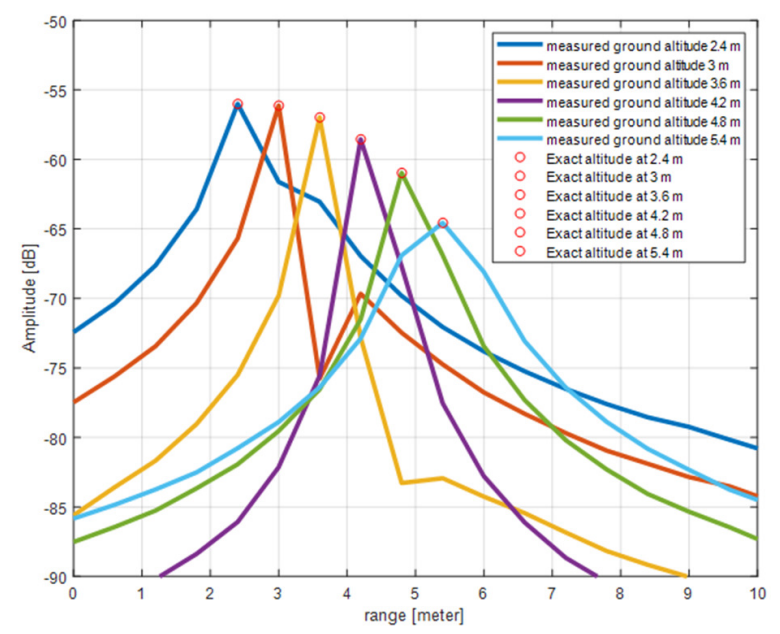

Fig. 2. Determining the flying ground level using UAV radar.

In the first scenario, the scattering field from constant circular trajectory flying pattern was simulated as shown in Figure 3, while snapshotting the received scattering field at 969 locations around the measurement domain to mimic the airborne UAV's collecting data. The measurement domain contains three cylinders placed 0.075 from the center at shown in Figure 4. Each cylinder has a diameter of $0.0375 \mathrm{~m}$ and a height of $0.05 \mathrm{~m}$. After the data of the single $2 \mathrm{GHz}$ frequency 
were stored at a scattering field vector in multiple snapshot locations, we started to calculate the dynamic Green's function based on the distance obtained from radar range at $500 \mathrm{MHz}$ bandwidth at L-band to be added into the matching filter matrix. Since the inversion is an ill-posed condition, the inversion between the scattering filed vector and the matching filter matrix has been done using the $\mathrm{CG}$ algorithm. The reconstructed xy-plane image of the measurement domain is shown in Figure 5 in which the pixel size is $0.00375 \mathrm{~m}$.

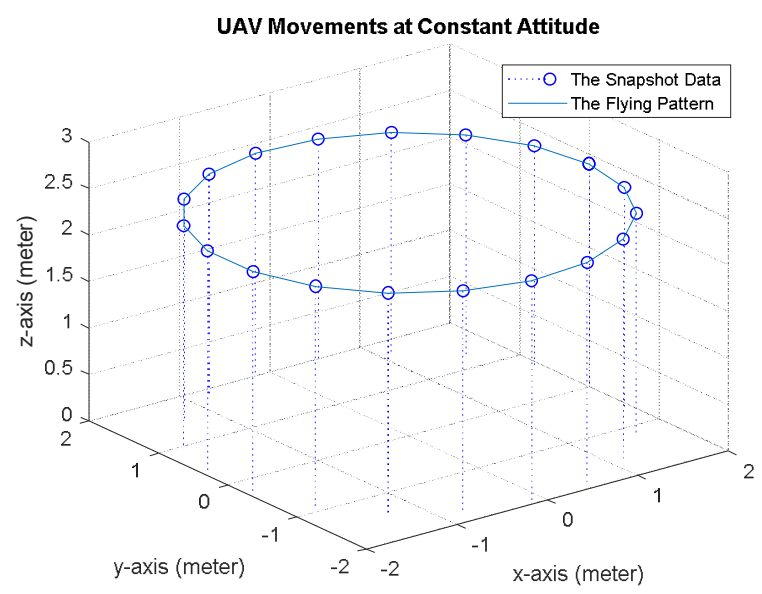

Fig. 3. UAV collecting data at constant flying attitude.

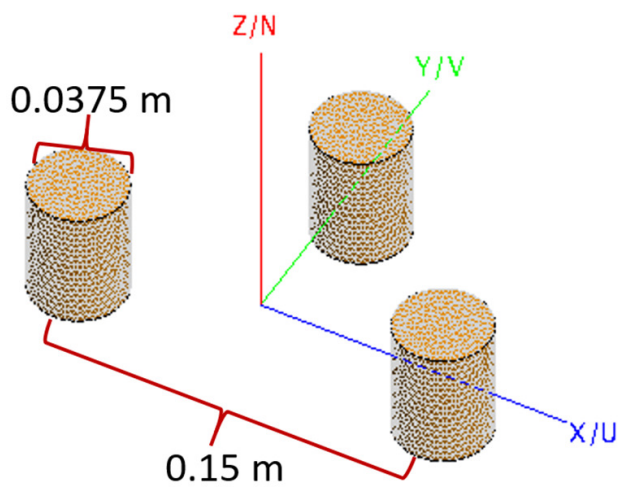

Fig. 4. Three cylinders at the measurement domain using FEKO simulation.

In the second scenario, we collected snapshot data at erratic flying pattern as shown in Figure 6. The snapshot data of the scattering field were collected at a circular trajectory from different flying levels at 969 different locations around the measurement domain. We used a multistatic scanning mode to apply different UAV scannings at the same time. The operation frequency is $2 \mathrm{GHz}$ with a wavelength of $15 \mathrm{~cm}$. The measurement domain contains five cylinders placed in the scene, as shown in Figure 7. Each cylinder has a diameter of $0.375 \mathrm{~m}$ spaced equally by $0.075 \mathrm{~m}$ at the $\mathrm{x}$-axis and $\mathrm{y}$-axis. The $\mathrm{x}-\mathrm{y}$ plane in Figure 8 is the cross-sectional of the $\mathrm{z}$-plane at 0 , and the pixel size is $0.00375 \mathrm{~m}$. The objects at the measurement domain appear as weak reflections due to the distance between the transmitters and the receivers, the varying UAV flying pattern, and the number of snapshot measurements, as shown in Figure 8 .

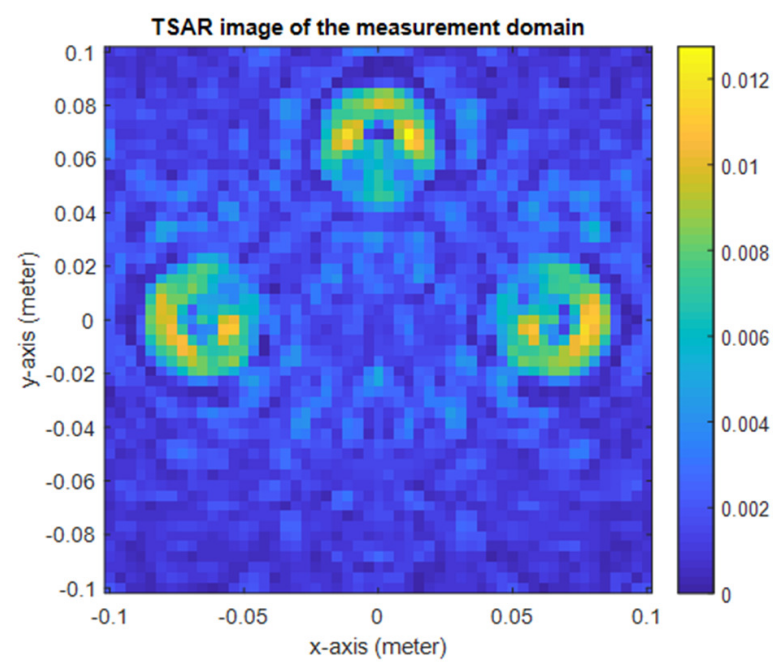

Fig. 5. The reconstructed TSAR of three cylinders at the measurement domain.

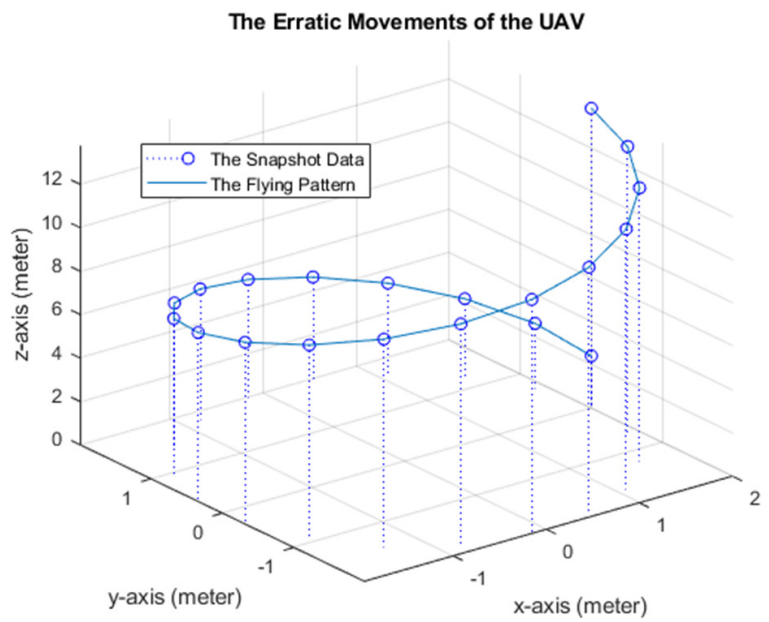

Fig. 6. The erratic flying pattern scenario of the UAV.

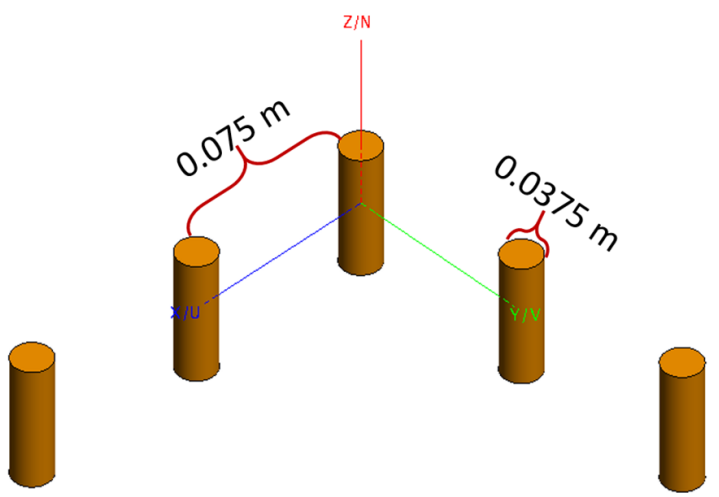

Fig. 7. Five cylinders at the measurement doamin using FEKO simulation.

After collecting the scattering field, the inversion process has been done using the $\mathrm{CG}$ algorithm to obtain the object function from the matching filter matrix that has been calculated due to each snapshot position and direction through the Green's function. The inversion produced the final 
reconstructed image. The final reconstructed image can be extended into a 3-D model image.

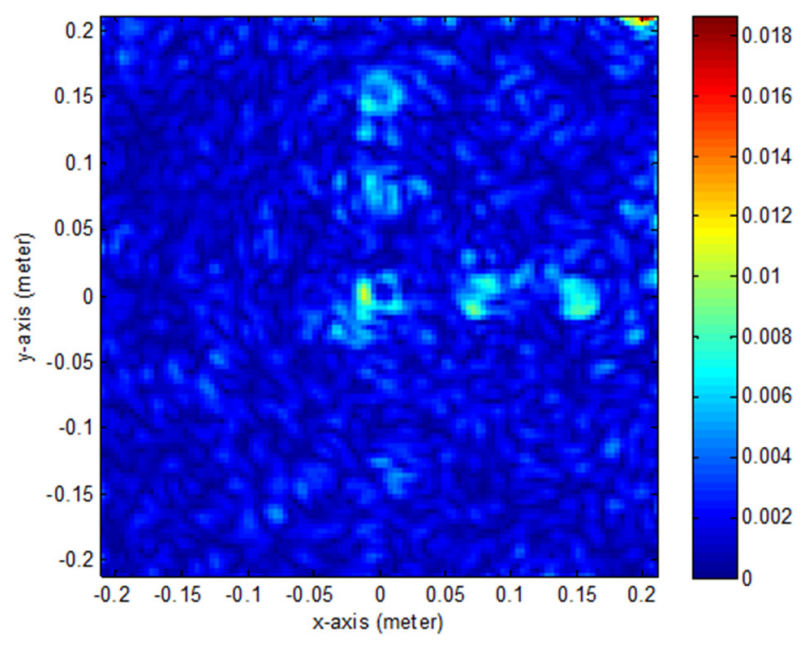

Fig. 8. The reconstructed TSAR image of the measurement domain.

\section{CONCLUSION}

In the Middle East there are more than 50,000 landmines buried randomly under the Saudi-Yemeni border, posing a fatal threat for the civilians. Unfortunately, most of the buried mines are nonmetallic, which are challenging to be detected in a large area by conventional methods. A large area needs to be prescreened for landmines before it becomes safe to use. Nevertheless, the synthesis array antenna is the output of a radar sensor onboard an aircraft to increase the airborne radar aperture length. The image formation algorithm generates the output image based on the collected signals, where the matched filter will apply autocorrelation to the input signal. Overall, SAR will treat the clutter as a processed signal to form an image. Appropriate SAR image requires high bandwidth and aperture parameters to obtain the desired resolution. On the other hand, soil losses degrade the penetrating depth based on the frequency and bandwidth for more underground sensing applications using SAR techniques. In the tomographic SAR mode, the data are collected as multistatic geometry exploiting the Spectral/Spatial Diversity. Furthermore, the multistatic decouple the relation between frequency forming the image and the radiated frequency. TSAR can be formed by using the ultranarrow band (UNB) at L-band to give more resolution than the conventional SAR, where NBU reduces the signal attenuation for more penetration depth. For UAV's TSAR, the measurement vector presents the collected signal in a multistatic mode. The matched filter is the electric field response at any flying location and direction, and the contrast vector (the object function) is the pixel weight. The idea based on exploit microwave signals to create an image of an object or prescreening underground. The operation frequency based on the applications (e.g. more microwave image resolution, less penetrating depth) where we are imaging based on tomography techniques. The tomographic SAR techniques solve the contrast function of an object or land (image) as an inverse problem considering the received signal with its response in the medium. For our application, we need less bandwidth to increase the penetrating depth and obtain higher resolution. The UAV's TSAR techniques were recording the aviation information and linked it to the recorded scattering field as discrete data. However, these methods exploit the Spectral/Spatial Diversity, where the soil losses are always a problem for penetrating signals, but UNB gives more degrees of freedom at radiation power level. For more applications, tomographic SAR can mount Unmanned Ground Vehicles (UGVs). Finally, TSAR applications can also extend to remote sensing applications.

\section{REFERENCE}

[1] L. Robledo, M. Carrasco, and D. Mery, "A survey of land mine detection technology," International Journal of Remote Sensing, vol. 30, no. 9, pp. 2399-2410, May 2009, doi: 10.1080/01431160802549435.

[2] K. Schreiner, "Landmine detection research pushes forward, despite challenges," IEEE Intelligent Systems, vol. 17, no. 2, pp. 4-7, Apr. 2002, doi: 10.1109/MIS.2002.999212.

[3] P. Gao and L. M. Collins, "A two-dimensional generalized likelihood ratio test for land mine and small unexploded ordnance detection," Signal Processing, vol. 80, no. 8, pp. 1669-1686, Aug. 2000, doi: 10.1016/S0165-1684(00)00100-6.

[4] R. Brooks and G. Di Chiro, "Principles of computer assisted tomography (CAT) in radiographic and radioisotopic imaging," Physics in Medicine and Biology, vol. 21, no. 5, pp. 689-732, Sep. 1976, doi: 10.1088/00319155/21/5/001.

[5] R. M. Mersereau and A. V. Oppenheim, "Digital reconstruction of multidimensional signals from their projections," Proceedings of the IEEE, vol. 62, no. 10, pp. 1319-1338, Oct. 1974, doi: 10.1109/PROC.1974.9625.

[6] D. C. Munson, J. D. O'Brien, and W. K. Jenkins, "A tomographic formulation of spotlight-mode synthetic aperture radar," Proceedings of the IEEE, vol. 71, no. 8, pp. 917-925, Aug. 1983, doi: 10.1109/PROC.1983.12698.

[7] A. C. Kak and M. Slaney, Principles of Computerized Tomographic Imaging. IEEE Press, 1988.

[8] M. D. Desai and W. K. Jenkins, "Convolution backprojection image reconstruction for spotlight mode synthetic aperture radar," IEEE Transactions on Image Processing, vol. 1, no. 4, pp. 505-517, Oct. 1992, doi: 10.1109/83.199920.

[9] O. Ponce et al., "Fully Polarimetric High-Resolution 3-D Imaging With Circular SAR at L-Band," IEEE Transactions on Geoscience and Remote Sensing, vol. 52, no. 6, pp. 3074-3090, Jun. 2014, doi: 10.1109/TGRS.2013.2269194.

[10] L. Wei, T. Balz, L. Zhang, and M. Liao, "A Novel Fast Approach for SAR Tomography: Two-Step Iterative Shrinkage/Thresholding," IEEE Geoscience and Remote Sensing Letters, vol. 12, no. 6, pp. 1377-1381, Jun. 2015, doi: 10.1109/LGRS.2015.2402124.

[11] S. Bertoldo, C. Lucianaz, M. Allegretti, O. Rorato, A. Prato, and G. Perona, "An Operative X-band Mini-radar Network to Monitor Rainfall Events with High Time and Space Resolution," Engineering, Technology \& Applied Science Research, vol. 2, no. 4, pp. 246-250, Aug. 2012.

[12] S. Bertoldo, C. Lucianaz, and M. Allegretti, "On the Use of a $77 \mathrm{GHz}$ Automotive Radar as a Microwave Rain Gauge," Engineering, Technology \& Applied Science Research, vol. 8, no. 1, pp. 2356-2360, Feb. 2018.

[13] C. Stringham, "GPU Processing for UAS-Based LFM-CW Stripmap SAR," ISPRS Journal of Photogrammetry and Remote Sensing, vol. 80, pp. 1107-1115, Dec. 2014, doi: 10.14358/PERS.80.12.1107.

[14] H. Sheng, K. Wang, X. Liu, and J. Li, "A fast raw data simulator for the stripmap SAR based on CUDA via GPU," in 2013 IEEE International Geoscience and Remote Sensing Symposium - IGARSS, Melbourne, VIC, Australia, Jul. 2013, pp. 915-918, doi: 10.1109/IGARSS.2013.6721309.

[15] L. Lo Monte, D. Erricolo, F. Soldovieri, and M. C. Wicks, "Radio Frequency Tomography for Tunnel Detection," IEEE Transactions on 
Geoscience and Remote Sensing, vol. 48, no. 3, pp. 1128-1137, Mar. 2010, doi: 10.1109/TGRS.2009.2029341.

[16] D. W. Paglieroni, D. H. Chambers, J. E. Mast, S. W. Bond, and N. Reginald Beer, "Imaging Modes for Ground Penetrating Radar and Their Relation to Detection Performance," IEEE Journal of Selected Topics in Applied Earth Observations and Remote Sensing, vol. 8, no. 3, pp. 1132-1144, Mar. 2015, doi: 10.1109/JSTARS.2014.2357718.

[17] M. Sato, "Principles of Mine Detection by Ground-penetrating Radar," in K. Furuta and J. Ishikawa, Eds., Anti-personnel Landmine Detection for Humanitarian Demining: The Current Situation and Future Direction for Japanese Research and Development. London, UK: Springer-Verlag, 2009

[18] J. Dula, A. Zare, D. Ho, and P. Gader, "Landmine classification using possibilistic K-nearest neighbors with wideband electromagnetic induction data," in Detection and Sensing of Mines, Explosive Objects, and Obscured Targets XVIII, Jun. 2013, vol. 8709, p. 87091F, doi: 10.1117/12.2016490.

[19] L. Carin, N. Geng, M. McClure, J. Sichina, and Lam Nguyen, "Ultrawide-band synthetic-aperture radar for mine-field detection," IEEE Antennas and Propagation Magazine, vol. 41, no. 1, pp. 18-33, Feb. 1999, doi: 10.1109/74.755021.

[20] Joel Andrieu et al., "Land mine detection with an ultra-wideband SAR system," presented at the Detection and Remediation Technologies for Mines and Minelike Targets VII, Orlando, FL, USA, Aug. 2002, vol. 4742, pp. 237-247, doi: 10.1117/12.479094.

[21] P. D. Gader, M. Mystkowski, and Yunxin Zhao, "Landmine detection with ground penetrating radar using hidden Markov models," IEEE Transactions on Geoscience and Remote Sensing, vol. 39, no. 6, pp. 1231-1244, Jun. 2001, doi: 10.1109/36.927446.

[22] A. Manandhar, P. A. Torrione, L. M. Collins, and K. D. Morton, "Multiple-Instance Hidden Markov Model for GPR-Based Landmine Detection," IEEE Transactions on Geoscience and Remote Sensing, vol. 53, no. 4, pp. 1737-1745, Apr. 2015, doi: 10.1109/TGRS.2014.2346954.

[23] O. Missaoui, H. Frigui, and P. Gader, "Land-Mine Detection With Ground-Penetrating Radar Using Multistream Discrete Hidden Markov Models," IEEE Transactions on Geoscience and Remote Sensing, vol. 49, no. 6, pp. 2080-2099, Jun. 2011, doi: 10.1109/TGRS.2010.2090886.

[24] J. MacDonald and J. R. Lockwood, Alternatives for Landmine Detection., Santa Monica, CA, USA: Rand, 2003.

[25] J. van den Heuvel and F. Fiore, "Simulation study of x-ray backscatter imaging of pressure-plate improvised explosive devices," in SPIE Defense, Security, and Sensing, Baltimore, Maryland, USA, Apr. 2012.

[26] H. Kasban, O. Zahran, S. M. Elaraby, M. El-Kordy, and F. E. Abd ElSamie, "A Comparative Study of Landmine Detection Techniques," Sensing and Imaging: An International Journal, vol. 11, no. 3, pp. 89112, Sep. 2010, doi: 10.1007/s1 1220-010-0054-x.

[27] L. Yujiri, S. W. Fornaca, B. I. Hauss, M. Shoucri, and S. Talmadge, "Detection of metal and plastic mines using passive millimeter waves," presented at the Detection and Remediation Technologies for Mines and Minelike Targets, Orlando, FL, United States, Apr. 1996, vol. 2765, doi: $10.1117 / 12.241235$.

[28] H. Ozturk et al., "Millimeter-wave detection of landmines," in Spie Defence, Security, and Sensing, Baltimore, Maryland, United States, May 2013, doi: 10.1117/12.2018026.

[29] M. E. A. Kanona, M. G. Hamza, A. G. Abdalla, and M. K. Hassan, "A Review of Ground Target Detection and Classification Techniques in Forward Scattering Radars," Engineering, Technology \& Applied Science Research, vol. 8, no. 3, pp. 3018-3022, Jun. 2018.

[30] E. Karpat, "CLEAN Technique to Classify and Detect Objects in Subsurface Imaging," International Journal of Antennas and Propagation, vol. 2012, Dec. 2012, doi: 10.1155/2012/917248, Art no. 1005000 .

[31] D. Carevic, "Kalman filter-based approach to target detection and targetbackground separtion in ground-penetrating radar data," presented at the Detection and Remediation Technologies for Mines and Minelike Targets IV, Orlando, FL, USA, Apr. 1999.

[32] G. Nadim, "Clutter reduction and detection of landmine objects in ground penetrating radar data using likelihood method," in $3 r d$
International Symposium on Communications, Control and Signal Processing, St Julians, Malta, Mar. 2008, pp. 98-106, doi: 10.1109/ISCCSP.2008.4537200.

[33] D. Carevic, "Clutter reduction and target detection in ground-penetrating radar data using wavelets," in Detection and Remediation Technologies for Mines and Minelike Targets IV, Orlando, FL, USA, Apr. 1999.

[34] D. Potin, E. Duflos, and P. Vanheeghe, "Landmines Ground-Penetrating Radar Signal Enhancement by Digital Filtering," IEEE Transactions on Geoscience and Remote Sensing, vol. 44, no. 9, pp. 2393-2406, Sep. 2006, doi: 10.1109/TGRS.2006.875356.

[35] A. C. Gurbuz, "Determination of Background Distribution for GroundPenetrating Radar Data," IEEE Geoscience and Remote Sensing Letters, vol. 9, no. 4, pp. 544-548, Jul. 2012, doi: 10.1109/LGRS.2011.2174137.

[36] M. C. Wicks, "RF Tomography with Application to Ground Penetrating Radar," in Conference Record of the Forty-First Asilomar Conference on Signals, Systems and Computers, Pacific Grove, CA, USA, Nov. 2007, pp. 2017-2022, doi: 10.1109/ACSSC.2007.4487591. 